\title{
Is the use of resveratrol in the treatment and prevention of obesity premature?
}

\author{
Wendy J. van der Spuy and Etheresia Pretorius* \\ Department of Anatomy, Faculty of Health Sciences, School of Medicine, University of Pretoria, \\ Pretoria, South Africa
}

\begin{abstract}
Obesity is a multi-faceted disease, predisposing sufferers to numerous co-morbidities such as epithelial dysfunction and insulin resistance which ultimately result in CVD. Visceral adipose tissue in particular is associated with inflammation due to the release of pro-inflammatory cytokines by adipocytes. Inflammation seems to be rather central in causing damage to endothelial cells as well as exerting negative effects on glucose metabolism, ultimately leading to insulin resistance. Resveratrol is a naturally occurring phenolic substance which has been found to display anti-inflammatory, vasoprotective and insulin-sensitising effects, among others. The popularity of resveratrol use is escalating in the treatment of various ailments including obesity in adults. The use of the substance in childhood obesity is, however, a worrying factor, as no studies have as yet been performed on adolescent animals and there is evidence of kidney toxicity of resveratrol and its metabolites at intake levels below those currently approved as safe. Another cause for concern is the uncertainty surrounding long-term, low-dose administration of the substance in humans. The supplement should thus not be recommended for use in the prevention and treatment of obesity until conclusive research is established on the safety of long-term usage of resveratrol in both children and adults.
\end{abstract}

Obesity: Inflammation: Cardiovascular disease: Resveratrol

\section{Introduction}

The amount of obese individuals in the world today is steadily reaching epidemic proportions. Importantly, obesity is not only an adult issue, but children are also increasingly affected. This increase in frequency of sufferers of obesity is mainly attributed to high-energy diets and low activity levels. Because of increased knowledge regarding the effects of obesity, many products and quick-fix solutions are currently on the market. Many of these products are alternative medicines or vitamins, typically seen as harmless and able to improve health. Particularly, products that are known to have antioxidant value claim to address obesity. The effects of some of these products on children and adults have not been adequately tested, and particularly in a condition like obesity, which is a health concern that is multi-faceted, could potentially have very negative outcomes.

Obesity is not a health complication on its own, but brings with it significant associated co-morbidities. These include CVD and heart attacks, type 2 diabetes mellitus, nonalcoholic fatty liver disease, insulin resistance ${ }^{(1,2)}$, hypertension, dyslipidaemia, impaired glucose homeostasis, sleep apnoea, accelerated skeletal and pubertal development (ageing processes), as well as cancer, orthopaedic disorders and psychological consequences ${ }^{(3-5)}$. Although many of these conditions only develop later in life, obese individuals are already setting the scene for their future health concerns when they are young.

The adipose tissue of visceral obesity in particular has been found to release pro-inflammatory cytokines. Inflammation seems to be central to the multitude of obesityrelated complications that shorten the lifespan of suffering individuals. Inflammation leads to endothelial dysfunction which ultimately results in CVD, either through atherogenesis or through more direct mechanisms. Inflammation also has a negative effect on glucose metabolism, leading to insulin resistance which is problematic either through resultant type 2 diabetes mellitus (impaired glucose homeostasis), or through causal relationships with endothelial dysfunction (insulin stimulates NO synthesis) which then eventually leads to CVD yet again. Insulin resistance is complicating in that it not only creates grounds for vascular effect impairment but also for metabolic impairment. Thus, obesity literally causes a cascade of interrelated health complications that ultimately shorten the lifespan of suffering individuals. For a schematic diagram of the inter-relationship of the obesity-related co-morbidities discussed here, see Fig. 1.

* Corresponding author: Dr E. Pretorius, fax + 2712319 2240, email resia.pretorius@up.ac.za 


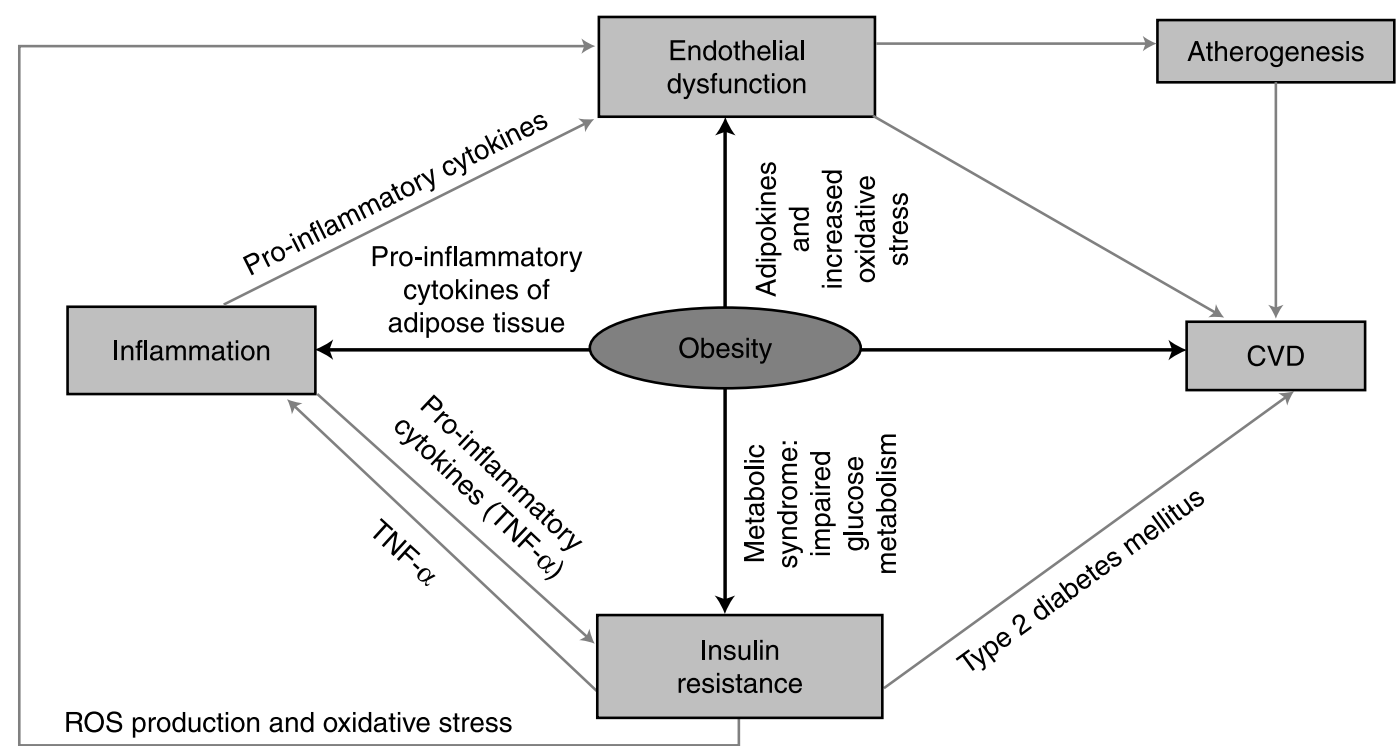

Fig. 1. Schematic diagram of the inter-relationship of most prominent co-morbidities of obesity. ROS, reactive oxygen species.

The link between obesity and endothelial dysfunction and insulin resistance is strengthened by the finding that endothelium-dependent vasodilation is impaired in proportion to insulin resistance and various indices of adiposity under baseline conditions ${ }^{(1,6)}$.

One of the antioxidants/anti-inflammatories that is now seen as a being advantageous in the treatment of obesity is resveratrol, and, although it is typically used by adults, there are no restrictions against its use by children. Resveratrol is a naturally occurring phenolic substance, present in a variety of plants as a defence mechanism against bacterial and other infections ${ }^{(5,7)}$ as well as environmental stressors ${ }^{(8)}$. A wide range of beneficial effects have been attributed to resveratrol, including antiinflammatory, anti-cancer, anti-lipid, anti-ageing, as well as vasoprotective effects ${ }^{(5,7)}$. The substance has been reported to elicit numerous cellular responses including cell cycle arrest, differentiation and apoptosis ${ }^{(9,10)}$. It also displays anti-leukaemic, antiviral and neuroprotective properties ${ }^{(10-13)}$. Resveratrol is able to function as an in vivo antioxidant ${ }^{(10,14)}$ and markedly reduces the risk of developing CHD; this property is probably due to its modulation of lipid metabolism and prevention of oxidation of the $\operatorname{LDL}^{(10,15)}$, as well as inhibition of eicosanoid production and platelet aggregation ${ }^{(10,16)}$. Additionally, resveratrol has been shown to inhibit several important enzymes involved in carcinogenesis ${ }^{(10,17,18)}$.

\section{Complications of obesity}

Obesity (Fig. 1) is characterised by an excess amount of adipose tissue. It has become recognised as an independent factor of cardiovascular risk, predisposing the obese individual to type 2 diabetes mellitus, hypertension, reduced endothelial function, as well as inducing insulin resistance ${ }^{(3,5)}$. Noteworthy is the fact that obesity is a component of the metabolic syndrome, of which insulin resistance is the hallmark. Metabolic risk factors associated with obesity are serum TAG elevations, low levels of HDL, elevated blood pressure, insulin resistance due to elevated glucose levels, as well as prothrombotic and pro-inflammatory states ${ }^{(1,19)}$.

\section{Inflammation}

Obesity is known to be accompanied by an inflammatory response (Fig. 1) in various tissues. Adipose tissue is being implicated, more and more, as a key regulator of inflammation through secretion of pro-inflammatory cytokines (i.e. TNF- $\alpha$ ) which seem to play a chief role in affecting both endothelial function and glucose metabolism $^{(1,20)}$. The negative influence on endothelial physiology may lead to the formation of atherosclerotic plaques ${ }^{(1)}$. Endothelial dysfunction and dysfunctional glucose metabolism (ultimately insulin resistance) seem to head the two main cascades which link obesity to CVD. In fact, growing evidence is pointing to a causative relationship between inflammation and insulin resistance, as TNF- $\alpha$ has been established to mediate insulin resistance as a result of obesity in a multitude of rodent obesity models ${ }^{(1,21)}$.

Resveratrol inhibits monocyte chemo-attractant protein secretion and gene transcription via down-regulation of TNF-induced transcription. It also reverses secretion and expression of atherogenic adipokines, actually reducing the size, density and mean area of atherosclerotic plaques, as well as the thickness of the vessel intima layer of atherosclerotic lesions ${ }^{(5,22)}$. Treatment with resveratrol, in addition to relieving glycerol-induced renal injury by suppressing the inflammatory process and by inhibiting lipid peroxidation $^{(5,23)}$, also significantly attenuates renal dysfunction and oxidative stress in diabetic rats ${ }^{(5,24)}$; therefore, assuming a significant anti-inflammatory role (Fig. 2) for resveratrol is plausible ${ }^{(5)}$

However, evidence has arisen showing an increase in both lipid peroxidation and DNA and protein carbonyl damage markers with resveratrol treatment in the kidneys, suggesting the possibility of kidney toxicity of resveratrol 


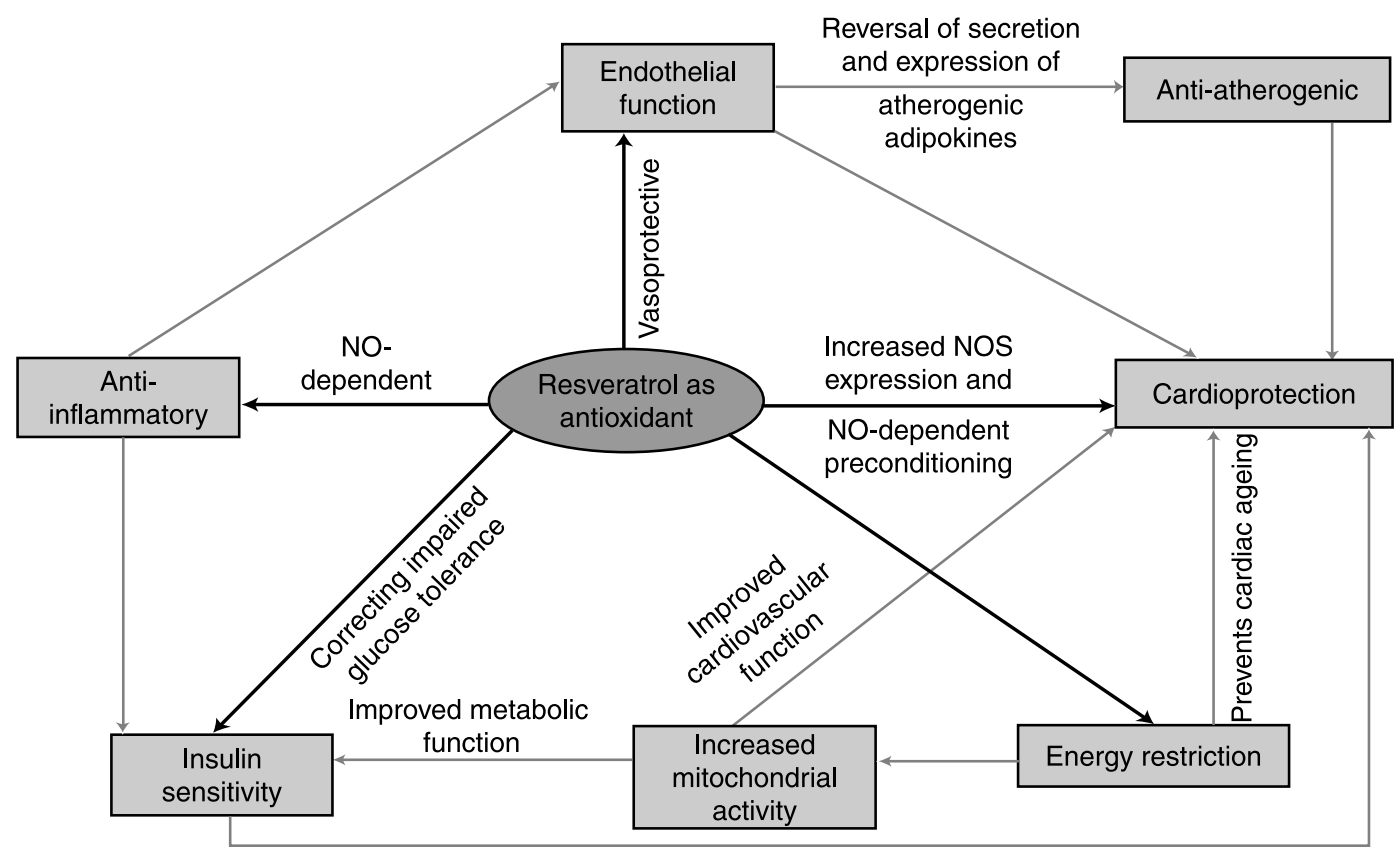

Fig. 2. Schematic diagram of benefits of resveratrol in prominent co-morbidities of obesity.

and its metabolites. Resveratrol forms extensive conjugated metabolites including glucuronides, sulfates and methylated products $^{(25-27)}$. Pharmacokinetic studies on acute resveratrol doses indicate significant distribution thereof to the kidneys ${ }^{(27,28)}$, with rapid excretion in urine ${ }^{(25,27,29)}$. These metabolites are capable of crossing glomerular membranes and accumulating in the kidney tubules where they may act as pro-oxidants or activate oxidative stress, as seen for various polyphenolic conjugates ${ }^{(27,30)}$. Wong et al. ${ }^{(27)}$ hypothesised that this may have led to the increase in lipid and protein damage observed in the kidneys of the resveratrol-treated mice in the study which they undertook. However, further in vivo studies on the biological activity of resveratrol metabolites are needed to investigate the chronic effects of low-dose resveratrol on the kidney.

Given the growing popularity of resveratrol as a supplement, the possibility of kidney damage is a cause for concern and warrants further investigation, especially before the supplement is recommended for usage in obesity treatment.

\section{Reduced endothelial function}

Vascular endothelial cells maintain cardiovascular homeostasis. Importantly, in addition to the physical barrier which they create, endothelial cells secrete a number of mediators that regulate platelet aggregation, coagulation, fibrinolysis and vessel tone ${ }^{(1)}$. There is strong evidence indicating an interaction between endothelium and the secretory proteins of adipocytes (adipokines), suggesting that the ability of adipokines to directly affect vascular homeostasis may be of importance in the developmental mechanism of CVD (Fig. 1) in obese patients ${ }^{(1,31)}$

Endothelium is a contributor to the regulation of blood pressure and flow, by releasing NO as well as other compounds which contribute to both vasodilation and vasoconstriction $^{(1)}$. NO is the major contributor to endothelium-dependent relaxation in conduit arteries ${ }^{(1,32)}$. Obesity has been shown to increase endothelium-dependent vasoconstriction in the absence of endothelial $\mathrm{NO}^{(5,33)}$.

It is the increased oxidative stress in accumulated fat which plays a major role in the induction of endothelial dysfunction. Endothelium thus loses its physiological properties which include the tendency to promote vasodilation, fibrinolysis, and its anti-aggregation properties ${ }^{(1)}$. NO has numerous important effects on the vasculature; these include the maintenance of basal tone by relaxing smooth muscle cells, inhibition of platelet adhesion, activation, secretion and aggregation, as well as the promotion of platelet disaggregation $^{(1,34)}$. Additionally, NO is a potent inhibitor of mechanisms ultimately leading to neointimal proliferation and atherosclerosis, through preventing endothelial leucocyte adhesion and smooth muscle cell migration and proliferation ${ }^{(1)}$.

Resveratrol has the ability to increase NO synthesis, which in turn functions as an in vivo antioxidant in the ischaemic-reperfused heart, brain or kidney, and lowers oxidative stress $^{(8,35)}$.

\section{Cardiovascular effects}

Resveratrol's protective mechanism (Fig. 2) includes its role as an intracellular antioxidant, anti-inflammatory agent, its ability to induce NO synthase (NOS) expression, as well as its ability to induce angiogenesis ${ }^{(8,12)}$. NO was thus identified to have a direct role in vasorelaxation when increased NOS activity was found in cultured pulmonary artery endothelial cells treated with resveratrol ${ }^{(36)}$, suggesting cardioprotection by resveratrol through affecting the expression of $\operatorname{NOS}^{(8)}$. 
Much evidence suggests that resveratrol-mediated cardioprotection is achieved by preconditioning ${ }^{(8,35)}$. Preconditioning cardioprotection is achieved by subjecting the heart to a therapeutic amount of stress, so as to disturb normal cardiovascular homeostasis and re-establish a modified homeostatic condition with increased cardiac defences that can withstand subsequent stress insult ${ }^{(8)}$. Resveratrol's pharmacological action for preconditioning of the heart takes place in an NO-dependent manner. The anti-inflammatory action itself of resveratrol seems to take place through an NO-dependent mechanism ${ }^{(8)}$.

Use of resveratrol improves post-ischaemic cardiac function regardless of the presence of glucose intake, and reduces the incidence of ventricular fibrillation and infarct size. Moreover, resveratrol increases GLUT-4 expression while reducing endothelin expression and cardiac apoptosis in ischaemic-reperfused hearts, also regardless of the absence of glucose intake, suggesting a direct protective effect on the heart ${ }^{(5,8,37,38)}$. GLUT-4 translocation is not involved in resveratrol's action, but the mechanism of action appears to lead to stimulation of the intrinsic activity of the GLUT-4 glucose transporters themselves ${ }^{(7)}$.

\section{Insulin resistance}

Insulin resistance (Fig. 1) is one of the pathophysiological mechanisms leading to diabetes mellitus ${ }^{(10,39)}$ and the state of resistance is attained through a decreased sensitivity or responsiveness of peripheral tissues to the metabolic action of insulin. This, together with the other components of the metabolic syndrome, is associated with altered endothelial function, which ultimately leads to $\mathrm{CVD}^{(1)}$. Insulin resistance occurs due to high glucose levels, and can contribute to reactive oxygen species production $^{(1,40)}$ (oxidative stress then plays a role in endothelial dysfunction). The insulin-resistant state is additionally characterised by an increase in pro-inflammatory cytokines such as TNF- $\alpha$, which may also contribute to hepatocellular injury ${ }^{(10,41)}$. The process leading to insulin resistance leads to long-term damage, dysfunction, and failure of various organs particularly the eyes, kidneys, nerves, heart and blood vessels ${ }^{(10,42)}$.

Resveratrol is capable of scavenging some intracellular reactive oxygen species ${ }^{(12)}$. Oral treatment with resveratrol significantly decreases the levels of glycosylated $\mathrm{Hb}$ (blood glucose indicator), suggesting that it may in fact prevent oxidative damage caused by the glycation reaction in diabetic conditions. Thus, resveratrol seems to be beneficial in preventing the pathogenesis of diabetic complications caused by impaired glucose metabolism ${ }^{(10)}$. Impaired glucose tolerance serves as a marker for insulin-resistant states and predicts vascular complications ${ }^{(10,43)}$. With resveratrol treatment, the impaired glucose tolerance observed in various groups of diabetic animals was corrected to near normal, strengthening the data on resveratrol's insulin-stimulatory (Fig. 2) effects ${ }^{(7,10)}$

Data from a study by Breen et al. ${ }^{(7)}$ indicate that resveratrol increases glucose uptake without stimulating the translocation of glucose transporters (i.e. GLUT-4) in skeletal muscle tissue. They demonstrated that resveratrol stimulates glucose uptake in skeletal muscle cells in a doseand time-dependent fashion, independent of insulin.

\section{Energy restriction}

The purpose of energy restriction is to retard several aspects of ageing; these include age-related mortality, tumorigenesis, as well as physiological decline and the establishment of age-related transcriptional profiles ${ }^{(44,45)}$. It is proposed that energy restriction confers health benefits by provoking an organismal defence response through mild stress ${ }^{(46,47)}$.

Animal studies have reported effects of energy restriction (Fig. 2) for resveratrol ${ }^{(2,5,38,45)}$, ultimately resulting in weight reduction, through prevention of abdominal obesity and TAG accumulation in hepatic cells, and improvement of lifespan of animals fed a high-energy diet. This weightreduction effect of resveratrol is speculated to be in part attributed to its effects on adipocytes ${ }^{(2,5,38)}$. Studies have reported a decrease in adipocyte size and decreased cell viability of adipocytes (both maturing and mature) through increasing apoptosis in these cells ${ }^{(5)}$.

Resveratrol mimics the effects of energy restriction to prevent cardiac ageing at both the transcriptional and functional levels. Additionally, mice on an energy-restricted diet or supplemented with resveratrol have improved insulin sensitivity (Fig. 2), as both these lower blood glucose levels without lowering plasma insulin ${ }^{(45)}$.

There is also a suggested up-regulation of gene expression regulating mitochondrial activity (Fig. 2) through resveratrol treatment as well as a proven increase in aerobic capacity. The increases in mitochondrial oxidative phosphorylation and aerobic capacity are associated with increased longevity, therefore improving animal survival time ${ }^{(5,48)}$.

Mitochondria are the principal energy sources of the cell that convert nutrients into energy through cellular respiration ${ }^{(49,50)}$. Compromised mitochondrial function has been linked to numerous diseases, including those of the metabolic and cardiovascular systems ${ }^{(50,51)}$. With impaired mitochondrial function, fatty acids are directed toward storage as opposed to oxidation. This may contribute to the lipid accumulation within muscle cells, which has been linked to insulin resistance in obesity and type 2 diabetes mellitus ${ }^{(50,52-54)}$. In line with this, resveratrol significantly improves both muscle oxidative capacity and sensitivity to insulin in high-energy-fed mice ${ }^{(50)}$.

Baur et al. ${ }^{(2)}$ hypothesised that resveratrol might shift the physiology of middle-aged mice on a high-energy diet towards that of mice on a standard diet and provide the associated health benefits of such a diet without having to reduce energy intake. After 6 months of treatment, they reported that there was a clear trend towards increased survival and insulin sensitivity. Also, it was found that resveratrol-treated mice on a high-energy diet displayed decreased organ pathology (liver, pancreas) when compared with untreated mice on a high-energy diet. The ability of resveratrol to improve motor function was also noted, indicating that the effects of resveratrol are not confined to the liver. Improvements in the morphology of the aortic elastic lamina were also apparent in resveratrol-treated mice. The livers of resveratrol-treated animals showed 
a considerable increase in the number of mitochondria when compared with untreated high-energy-fed mice, and were not significantly different from mice on a standard diet. The data collected by this group demonstrate quite clearly that resveratrol can alleviate the negative impact of a high-energy diet on the overall health and lifespan of middle-aged mice.

The findings of this group ${ }^{(2)}$ showed that resveratrol does indeed shift the physiology of mice consuming high-energy diets towards that of mice on a standard diet. Resveratrol was also shown to modulate known longevity pathways and improve general health, including survival, motor function, insulin sensitivity, organ pathology, and mitochondrial number. These changes occurred, notably, without significant reduction in body weight.

It is, however, important to note that all experimental treatments have thus far been administered to middle-aged animal models; therefore, it has not yet been ascertained what the effects of resveratrol treatment would be on young animals. Although the present review focuses on the general effects of resveratrol on obesity, it cannot be ignored that children may prove to be more prone than adults to its potentially harmful effects. Thus, before any recommendation is made for the use of resveratrol in the treatment or prevention of obesity, studies will have to specifically test the product on adolescent animals, as well as compare differences in toxicity data with those of experiments performed on adult animals.

\section{Multi-faceted effects of resveratrol}

Resveratrol seems to present with two faces. It protects cells by potentiating a survival signal, but selectively kills cancer cells. It behaves as an antioxidant, yet can induce redox signalling. It is an anti-proliferative agent for cancer, inducing apoptosis in tumour cells and sensitising cancer cells by inhibiting cell survival signal transduction and anti-apoptotic pathways. In contrast, it also triggers a survival signal in ischaemic tissue by inducing antiapoptotic genes and blocking apoptosis in the ischaemic heart. At low doses, resveratrol stimulates angiogenesis, but at higher doses it blocks the angiogenic response. At low concentrations, resveratrol scavenges reactive oxygen species, but at higher concentrations, it behaves like a pro-oxidant. Low concentrations of resveratrol are quite sufficient for cardiovascular preconditioning, whereas higher concentrations and chronic use thereof may, in fact, exert toxic effects in the heart and other organs ${ }^{(8)}$.

In general, however, adverse effects of resveratrol have not been documented in humans, and a recommended dosage of $5-10 \mathrm{mg} / \mathrm{d}$ is suggested to be safe after a review of toxicological literature for resveratrol by the National Institute of Environmental Health Sciences ${ }^{(55)}$. However, the elevation in oxidative damage observed in the kidneys of mice in the study by Wong et al. ${ }^{(27)}$ over an extended period took place at levels well below that for which toxicity has previously been reported ${ }^{(2,56,57)}$. This raises concern regarding long-term, low-dose administration of resveratrol ${ }^{(27)}$. Thus, it would not be considered wise to use resveratrol at this time for the prevention or treatment of obesity, especially in children, as long-term toxicity could in fact cause more detrimental harm to the systems of suffering individuals than currently anticipated.

\section{Conclusion}

Obesity is a growing problem worldwide, and treatment potentials are constantly under scrutiny. There is a prevalence of inflammation in obese patients, and this brings with it a cascade of problems including endothelial dysfunction and insulin resistance, ultimately resulting in cardiovascular complications. Resveratrol is a phenolic substance in which much interest has been placed. The substance is anti-inflammatory, vasoprotective, and increases glucose uptake, thus improving insulin sensitivity. These effects are the ones most applicable to obesity prevention and treatment. However, the use of resveratrol over the long term even in low doses has not yet been conclusively ascertained. Additionally, there are reports of kidney toxicity of resveratrol and its metabolites even at concentration levels currently approved by the National Institute of Environmental Health Sciences. It is thus wise not to recommend the use of resveratrol in the treatment of obesity, especially childhood obesity, as all experimental data available are from studies utilising middle-aged animal models, unless and until the safety of resveratrol and its metabolites have been scrutinised to a definitive level.

\section{Acknowledgements}

The authors contributed equally to the present paper and thank the National Research Foundation of South Africa (NRF) for funding E. P. (Indigenous Knowledge Systems (FA2004033100004)).

The authors have no conflict of interest and the authors have no financial interests in the products reviewed in this paper.

\section{References}

1. Avogaro A \& de Kreutzenberg SV (2005) Mechanisms of endothelial dysfunction in obesity. Clin Chim Acta 360, 9-26.

2. Baur JA, Pearson KJ, Price NL, et al. (2006) Resveratrol improves health and survival of mice on a high calorie diet. Nature 444, 337-342.

3. Wellman NS \& Friedberg B (2002) Causes and consequences of adult obesity: health, social and economic impacts in the United States. Asia Pac J Clin Nutr 11, Suppl. 8, s705-s709.

4. Levy LZ \& Petty K (2008) Childhood obesity prevention: compelling challenge of the twenty-first century. Early Child Dev Care 178, 609-615.

5. Naderali EK (2009) Obesity and cardiovascular dysfunction: a role for resveratrol? Obes Res Clin Pract 3, 45-52.

6. De Jongh RT, Serne EH, Ilserman RG, et al. (2004) Impaired microvascular function in obesity: implications for obesityassociated microangiopathy, hypertension, and insulin resistance. Circulation 109, 2529-2535.

7. Breen DM, Sanli A, Giacca A, et al. (2008) Stimulation of muscle cell glucose uptake by resveratrol through sirtuins and AMPK. Biochem Biophys Res Commun 374, 117-122.

8. Das KD \& Maulik N (2006) Resveratrol in cardioprotection: a therapeutic promise of alternative medicine. Mol Interventions 6, 36-47. 
9. Joe AK, Liu H, Suzui M, et al. (2002) Resveratrol induces growth inhibition, S-phase arrest, apoptosis, and changes in biomarker expression in several human cancer cell lines. Clin Cancer Res 8, 893-903.

10. Palsamy P \& Subramanian S (2008) Resveratrol, a natural phytoalexin, normalizes hyperglycemia in streptozotocinnicotinamide induced experimental diabetic rats. Biomed Pharmacother 62, 598-605.

11. Virgili M \& Contestabile A (2000) Partial neuroprotection of in vivo excitotoxic brain damage by chronic administration of the red wine antioxidant agent, trans-resveratrol in rats. Neurosci Lett 281, 123-126.

12. Bhat KP, Kosmeder JW II \& Pezzuto JM (2001) Biological effects of resveratrol. Antioxid Redox Signal 3, 1041-1064.

13. Gao X, Xu YX, Divine G, et al. (2002) Disparate in vitro and in vivo antileukemic effects of resveratrol, a natural polyphenolic compound found in grapes. J Nutr 132, 2076-2081.

14. Das DK, Sato M, Ray PS, et al. (1999) Cardioprotection of red wine: role of polyphenolic antioxidants. Drugs Exp Clin Res 25, 115-120.

15. Frankel EN, Waterhouse AL \& Kinsella JE (1993) Inhibition of human LDL oxidation by resveratrol. Lancet 341, 1103-1104.

16. Pace-Asciak CR, Hahn S, Diamandis EP, et al. (1995) The red wine phenolics trans-resveratrol and quercetin block human platelet aggregation and eicosanoid synthesis: implications for protection against coronary heart disease. Clin Chim Acta 235, 207-219.

17. Fontecave M, Lepoivre M, Elleingand E, et al. (1998) Resveratrol, a remarkable inhibitor of ribonucleotide reductase. FEBS Letters 421, 277-279.

18. Chun YJ, Kim MY \& Guengerich FP (1999) Resveratrol is a selective human cytochrome P450 1A1 inhibitor. Biochem Biophys Res Commun 262, 20-24.

19. Stern MP (1995) Diabetes and cardiovascular disease. The 'common soil' hypothesis. Diabetes 44, 369-374.

20. Yudkin JS (2003) Adipose tissue, insulin action and vascular disease: inflammatory signals. Int J Obes Relat Metab Disord 27, Suppl. 3, S25.

21. Hotamisligil GS (2003) Inflammatory pathways and insulin action. Int J Obes Relat Metab Disord 27, Suppl. 3, S53.

22. Penumathsa SV, Koneru S, Samuel SM, et al. (2008) Strategic targets to induce neovascularization by resveratrol in hypercholesterolemic rat myocardium: role of caveolin-1, endothelial nitric oxide synthase, hemoxygenase-1, and vascular endothelial growth factor. Free Radic Biol Med 45, $1027-1034$.

23. De Jesus Soares T, Volpini RA, Francescato HD, et al. (2007) Effects of resveratrol on glycerol-induced renal injury. Life Sci 81, 647-656.

24. Sharma S, Anjaneyulu M, Kulkarni SK, et al. (2006) Resveratrol, a polyphenolic phytoalexin, attenuates diabetic nephropathy in rats. Pharmacology 76, 69-75.

25. Meng X, Maliakal P, Lu H, et al. (2004) Urinary and plasma levels of resveratrol and quercetin in humans, mice, and rats after ingestion of pure compounds and grape juice. J Agric Food Chem 52, 935-942.

26. Wenzel E, Soldo T, Erbersdobler H, et al. (2005) Bioactivity and metabolism of trans-resveratrol orally administered to Wistar rats. Mol Nutr Food Res 49, 482-494.

27. Wong YT, Gruber J, Jenner AM, et al. (2009) Elevation of oxidative damage biomarkers during aging in F2 hybrid mice: protection by chronic oral intake of resveratrol. Free Radic Biol Med 46, 799-809.
28. Bertelli A, Bertelli AA, Gozzini A, et al. (1998) Plasma and tissue resveratrol concentrations and pharmacological activity. Drugs Exp Clin Res 24, 133-138.

29. Walle T, Hsieh F, DeLegge MH, et al. (2004) High absorption but very low bioavailability of oral resveratrol in humans. Drug Metab Dispos 32, 1377-1382.

30. Monks TJ \& Lau SS (1998) The pharmacology and toxicology of polyphenolic glutathione conjugates. Annu Rev Pharmacol Toxicol 38, 229-255.

31. Caballero AE (2003) Endothelial dysfunction in obesity and insulin resistance: a road to diabetes and heart disease. Obes Res 11, 1278-1289.

32. Hwa JJ, Ghibaudi L, Williams P, et al. (1994) Comparison of acetylcholine-dependent relaxation in large and small arteries of rat mesenteric vascular bed. Am J Physiol Heart Circ Physiol 266, H952-H958.

33. Steinberg HO, Chacker H, Leaming R, et al. (1996) Obesity/insulin resistance is associated with endothelial dysfunction: implications for the syndrome of insulin resistance. J Clin Invest 97, 2601-2610.

34. Anderson TJ (2003) Nitric oxide, atherosclerosis and the clinical relevance of endothelial dysfunction. Heart Fail Rev 8, 71-86.

35. Hattori R, Otani H, Maulik N, et al. (2002) Pharmacological preconditioning with resveratrol: role of nitric oxide. Am J Physiol Heart Circ Physiol 282, H1988-H1995.

36. Klinge CM, Risinger KE, Watts MB, et al. (2003) Estrogenic activity in white and red wine extracts. J Agric Food Chem 51, 1850-1857.

37. Sato M, Maulik G, Bagchi D, et al. (2000) Myocardial protection by protykin, a novel extract of trans-resveratrol and emodin. Free Radic Res 32, 135-144.

38. Lekli I, Szabo G, Juhaz B, et al. (2008) Protective mechanisms of resveratrol against ischemia-reperfusioninduced damage in hearts obtained from Zucker obese rats: the role of GLUT-4 and endothelin. Am J Physiol Heart Circ Physiol 294, H859-H866.

39. McCarthy MI \& Hattersley AT (2001) Molecular diagnosis in monogenic and multifactoral forms of type 2 diabetes. Expert Rev Mol Diagn 1, 403-412.

40. Inoguchi T, Li P, Umeda F, et al. (2000) High glucose level and free fatty acid stimulate reactive oxygen species production through protein kinase $\mathrm{C}$-dependent activation of NAD $(\mathrm{P}) \mathrm{H}$ oxidase in cultured vascular cells. Diabetes 49, $1939-1945$

41. Grove J, Daly AK, Bassendine MF, et al. (1997) Association of a tumor necrosis factor promoter polymorphism with susceptibility to alcoholic steatohepatitis. Hepatology 26, $143-146$.

42. Schuster DP \& Duvuuri V (2002) Diabetes mellitus. Clin Podiatr Med Surg 19, 79-107.

43. Tominaga M, Eguchi H, Manaka H, et al. (1999) Impaired glucose tolerance is a risk factor for cardiovascular disease, but not impaired fasting glucose. The Funagata Diabetes Study. Diabetes Care 22, 920-924.

44. Lee CK, Klopp RG, Weindruch R, et al. (1999) Gene expression profile of aging and its retardation by caloric restriction. Science 285, 1390-1393.

45. Barger JL, Kayo T, Vann JM, et al. (2008) A low dose of resveratrol partially mimics caloric restriction and retards aging parameters in mice. PLOS ONE 3, e2264.

46. Anderson RM, Bitterman KJ, Wood JG, et al. (2003) Nicotinamide and Pnc1 govern lifespan extension by calorie restriction in S. cerevisiae. Nature 423, 181-185.

47. Howitz KT, Bitterman KJ, Cohen HY, et al. (2003) Small molecule activators of sirtuins extend Saccharomyces cerevisiae lifespan. Nature 425, 191-196. 
48. Dasgupta B \& Milbrandt J (2007) Resveratrol stimulates AMP kinase activity in neurons. Proc Natl Acad Sci U S A 104, 7217-7222.

49. Wallace DC (2005) A mitochondrial paradigm of metabolic and degenerative diseases, aging, and cancer: a dawn for evolutionary medicine. Annu Rev Genet 39, 359-407.

50. Lagouge M, Argman C, Gerhart-Hines Z, et al. (2006) Resveratrol improves mitochondrial function and protects against metabolic disease by activating SIRT1 and PGC- $1 \alpha$. Cell 127, 1109-1122.

51. Petersen KF, Befroy D, Dufour S, et al. (2003) Mitochondrial dysfunction in the elderly: possible role in insulin resistance. Science 300, 1140-1142.

52. Virkamaki A, Korsheninnikova E, Seppala-Lindroos A, et al. (2001) Intramyocellular lipid is associated with resistance to in vivo insulin actions on glucose uptake, antilipolysis, and early insulin signaling pathways in human skeletal muscle. Diabetes 50, 2337-2343.

53. Patti ME, Butte AJ, Crunkhorn S, et al. (2003) Coordinated reduction of genes of oxidative metabolism in humans with insulin resistance and diabetes: potential role of PGC1 and NRF1. Proc Natl Acad Sci U S A 100, 8466-8471.

54. Petersen KF, Dufour S, Befroy D, et al. (2004) Impaired mitochondrial activity in the insulin-resistant offspring of patients with type 2 diabetes. $N$ Engl J Med 350, 664-671.

55. Haneke KE, Carson BL, Gregorio CA, et al. (2002) Review of Toxicological Literature for Resveratrol Prepared for Scott Masten. National Institute of Environmental Health Sciences (NIEHS). Submitted by Integrated Laboratory Systems, Inc., through NIEHS contract N01ES-6540. Research Triangle, NC: NIEHS. http://ntp. niehs.nih.gov/ntp/htdocs/Chem_Background/ExSumPDF/ resveratrol.pdf

56. Juan ME, Vinardell MP \& Planas JM (2002) The daily oral administration of high doses of trans-resveratrol to rats for 28 days is not harmful. J Nutr 132, 257-260.

57. Crowell JA, Korytko PJ, Morrissey RL, et al. (2004) Resveratrol-associated renal toxicity. Toxicol Sci 82, 614-619. 